\title{
Growth-related changes in intracellular spermidine and its effect on efflux pump expression and quorum sensing in Burkholderia pseudomallei
}

\author{
Correspondence \\ Kim Lee Chua \\ bchckl@nus.edu.sg
}

Received 18 July 2009

Revised 9 December 2009

Accepted 21 December 2009

\author{
Ying Ying Chan and Kim Lee Chua
}

\author{
Department of Biochemistry, Yong Loo Lin School of Medicine, National University of Singapore, \\ 8 Medical Drive, 117597, Singapore
}

\section{INTRODUCTION}

Melioidosis is a serious and potentially fatal septicaemic disease caused by the aerobic Gram-negative soil bacillus Burkholderia pseudomallei. The disease is endemic to southeast Asia and northern Australia, with sporadic cases occurring in other countries within latitudes $20^{\circ} \mathrm{N}$ and $20^{\circ} \mathrm{S}$ (Cheng \& Currie, 2005). Therapeutic options are limited, as the pathogen is intrinsically resistant to many commonly used antibiotics, including penicillins, first and second generation cephalosporins, aminoglycosides, rifamycins and macrolides, and relapses are common (Cheng \& Currie, 2005; White, 2003). Multidrug effux pumps of the resistance-nodulation-division (RND) family, such as BpeAB-OprB, AmrAB-OprA and BpeEF-OprC, are responsible for its resistance to aminoglycosides, macrolides, trimethoprim and chloramphenicol (Chan et al., 2004; Kumar et al., 2006; Moore et al., 1999). RND pumps also extrude diverse non-antibiotic substrates, including acylhomoserine lactones (AHLs), which are involved in quorum

Abbreviations: $\mathrm{AHL}$, acylhomoserine lactone; C8HSL, N-octanoylhomoserine lactone; C10HSL, $N$-decanoyl-homoserine lactone; EMSA, electrophoretic mobility shift assay; 3-hydroxy-C8HSL, N-3-hydroxyoctanoyl-homoserine lactone; 3-hydroxy-C1OHSL, N-3-hydroxy-decanoylhomoserine lactone; 3-oxo-C10HSL, 3-oxo-decanoyl-homoserine lactone; 3-oxo-C14HSL, N-3-oxo-tetradecanoyl-homoserine lactone; RND family, resistance-nodulation-division family; RP-HPLC, reversed-phase HPLC. sensing (Chan et al., 2007; Pearson et al., 1999). B. pseudomallei that lacks the BpeAB-OprB function is defective in quorum sensing, attenuated in virulence and also produces less biofilm (Chan \& Chua, 2005).

We investigated several intermediates and products of the methionine utilization pathway involved in the synthesis of AHLs and polyamines while trying to identify other physiological substrates of the BpeAB-OprB efflux pump. Putrescine and spermidine, the predominant polyamines in Gram-negative bacteria, are known to influence gene expression either by stimulating the activity of RNA polymerase or by stabilizing ribosomal structure and modulating translational fidelity (Huang et al., 1990; Igarashi \& Kashiwagi, 2000; Tabor \& Tabor, 1985). They affect translation by interacting directly with negatively charged DNA or RNA (Yoshida et al., 1999). They also serve as free radical scavengers to protect DNA from oxidative damage (Ha et al., 1998; Khan et al., 1992). Polyamines protect Escherichia coli against hydrogen peroxide-induced oxidative stress by inducing $\operatorname{oxy} R$ and rpoS gene expression, and increase its survival under extremely acid conditions, a property deemed essential for successful colonization of the mammalian host (Jung \& Kim, 2003a, b).

Apart from ensuring optimal growth, too many free polyamines are toxic to the bacterium because they can inhibit protein synthesis, especially during stationary phase 
and at low temperatures (Fukuchi et al., 1995; Limsuwun \& Jones, 2000). Hence, a balance between polyamine synthesis and catabolism is needed. Spermidine is synthesized from putrescine by spermidine synthase, which is strongly inhibited by compounds such as dicyclohexylamine and methylthiopropylamine (Pegg et al., 1983). Spermidine is also acetylated by spermidine acetyltransferase to $\mathrm{N}$-acetylspermidine, an inert and less toxic form, and bacteria that lack the acetyltransferase accumulate spermidine during the stationary phase and have reduced viability due to inhibition of protein synthesis (Seiler, 1987; Fukuchi et al., 1995).

Exogenous spermidine added to culture medium increases the resistance of Pseudomonas aeruginosa to aminoglycosides and quinolones, although no known efflux system was found to be significantly upregulated using DNA microarray analysis (Kwon \& Lu, 2006). In this study, we tracked the changes in intracellular levels of spermidine and $\mathrm{N}$-acetylspermidine during growth of $B$. pseudomallei KHW and describe the effect of such changes on the expression of the BpeAB-OprB pump, which is involved in the efflux of mostly $\mathrm{N}$-acetylspermidine. We also demonstrate the therapeutic potential of spermidine synthase inhibitors as antimicrobial agents that can attenuate quorum sensing, and reduce the swimming motility of and the formation of biofilms by B. pseudomallei KHW.

\section{METHODS}

Bacteria and plasmids. Wild-type $B$. pseudomallei KHW and its isogenic bpeAB deletion mutant, KHW $\triangle b p e A B$, as well as the $b p e A-$ lacZ reporter plasmid $\mathrm{pCYYbpeAB}$, have been described previously (Chan et al., 2004). B. pseudomallei was cultured under aerobic conditions at $37{ }^{\circ} \mathrm{C}$ in $\mathrm{AB}$ minimal medium supplemented with $0.2 \%$ (w/v) glucose and $0.2 \%$ casamino acids (Clark \& Maaløe, 1967), or in Luria-Bertani (LB) medium for the motility and biofilm assays. Kanamycin and tetracycline were used at final concentrations of 200 and $25 \mu \mathrm{g} \mathrm{ml}{ }^{-1}$, respectively. The above antibiotics and the spermidine synthase inhibitors dicyclohexylamine and methylthiopropylamine were purchased from Sigma-Aldrich.

Extraction and measurement of polyamines. Wild-type $B$. pseudomallei KHW and the bpeABoprB mutant were cultured in $\mathrm{AB}$ medium containing $0.2 \%(\mathrm{w} / \mathrm{v})$ glucose alone, without casamino acids. A $10 \mathrm{ml}$ volume of $\mathrm{AB}$ medium was inoculated with $200 \mu \mathrm{l}$ of overnight bacterial culture and incubated at $37{ }^{\circ} \mathrm{C}$, shaken at 100 r.p.m. After $0.5,6,10,14$ and $24 \mathrm{~h}$, a volume of culture equivalent to $1 \mathrm{OD}_{600}$ unit of cells $\left(\sim 10^{9}\right.$ cells $)$ was removed to extract polyamines using the procedure described by Jung \& Kim (2003b). One $\mathrm{OD}_{600}$ unit of cells is equivalent to $5.6 \mathrm{mg}$ wet weight of $B$. pseudomallei cells or $50 \mu \mathrm{g}$ total protein. The cells and supernatant were separated by centrifugation at 3000 r.p.m. for $15 \mathrm{~min}$. Intracellular polyamines were extracted from the cell pellet. After washing twice with $3 \mathrm{ml}$ PBS, the cell pellet was resuspended in $2 \mathrm{ml}$ $5 \%$ perchloric acid (Sigma) and incubated on ice for $1 \mathrm{~h}$ to lyse the cells. The cell debris was removed by centrifugation at $8000 \mathrm{~g}$ for $10 \mathrm{~min}$ at $4{ }^{\circ} \mathrm{C}$, and $1 \mathrm{ml} 2 \mathrm{M} \mathrm{NaOH}$ and $10 \mu \mathrm{l}$ benzoyl chloride were added to the cleared lysate for $40 \mathrm{~min}$ at $30{ }^{\circ} \mathrm{C}$ to derivatize the polyamines. Benzoylation caused the polyamines to absorb UV light at $254 \mathrm{~nm}$ and to be detected by reversed-phase HPLC (RP-HPLC) analysis. The benzoylation was stopped by adding $2 \mathrm{ml}$ saturated
$\mathrm{NaCl}$, and the derivatized polyamines were extracted with $2 \mathrm{ml}$ diethyl ether. The organic layer (top) was collected and dried. Polyamines in the culture supernatant were similarly benzoylated and extracted using diethyl ether. The dried extracts were each solubilized in $0.1 \mathrm{ml}$ methanol and filtered through a $0.45 \mu \mathrm{m}$ pore-size filter, and $20 \mu \mathrm{l}$ of the filtrate was applied onto a $200 \mathrm{~mm} \times 4.6 \mathrm{~mm} \mathrm{C} \mathrm{C}_{18}$ column (Agilent series 1100 Hypersil ODS, particle size $5 \mathrm{~mm}$ ). The polyamines were eluted as described by Hwang et al. (1997) using $50 \%(\mathrm{v} / \mathrm{v})$ methanol in water for $0.5 \mathrm{~min}$, followed by a linear gradient from $50 \%(\mathrm{v} / \mathrm{v})$ to $85 \%(\mathrm{v} / \mathrm{v})$ methanol in water for $6.5 \mathrm{~min}$ at a flow rate of $0.8 \mathrm{ml} \mathrm{min}^{-1}$ (Hwang et al., 1997). This was followed by an isocratic profile at $85 \%(\mathrm{v} / \mathrm{v})$ for $5 \mathrm{~min}$, and then a decrease over $2 \mathrm{~min}$ to $50 \%(\mathrm{v} / \mathrm{v})$ methanol in water at $0.8 \mathrm{ml} \mathrm{min}^{-1}$. Peaks corresponding to the polyamines were detected using absorption spectrophotometry at a wavelength of $254 \mathrm{~nm}$. Two millilitre volumes of the polyamine standards (ranging from 12.5 to $200 \mathrm{nM}$ of putrescine, spermine, spermidine and $\mathrm{N}$-acetylspermidine) (Sigma) were also benzoylated using $1 \mathrm{ml} 2 \mathrm{M} \mathrm{NaOH}$ and $10 \mu$ l benzoyl chloride, and extracted with diethyl ether. The dried extract was also solubilized in $0.1 \mathrm{ml}$ methanol, and $20 \mu \mathrm{l}$ was injected into the HPLC column. The retention times for putrescine, spermine, spermidine and $\mathrm{N}$-acetylspermidine were $7.3,10.1,9.5$ and $11.1 \mathrm{~min}$, respectively. The experiments were conducted in triplicate and the amounts of intracellular polyamines were expressed as pmol per $10^{9}$ cells (or 1 $\mathrm{OD}_{600}$ unit), while the concentrations of polyamines in the extracellular medium were expressed in $\mathrm{nM}$.

Electrophoretic mobility shift assays (EMSAs). A DNA fragment (868 bp) carrying the promoter sequences of both bpeABoprB and bpeR was amplified by PCR using the primer pair AcrABpro (5' CTTCCTCCTCGTGCGTCTGGC-3') and AcrA5'R (5'-GGCCACCCGCATCGTCGTA-3'). The DNA was cut using AvaI into two fragments, a $479 \mathrm{bp}$ bpeABoprB promoter fragment and a $389 \mathrm{bp}$ $b p e R$ promoter fragment. Each fragment was recovered after gel electrophoresis in 1.2\% agarose using the GeneClean II kit (Bio 101). Recombinant BpeR repressor protein was expressed in E. coli M15[pREP4] harbouring the expression vector pQE30 carrying the full-length $b p e R$, and was purified under native conditions according to the manufacturer's protocol (Qiagen). EMSA was performed as described by Fried and Crothers (1981). The repressor-DNA binding reaction $(10 \mu \mathrm{l})$ consisted of $1 \mu \mathrm{g}$ DNA fragment, $1 \mu \mathrm{g}$ purified BpeR, $1 \mu \mathrm{g}$ poly-dIdC and $100 \mu \mathrm{g}$ BSA in binding buffer $(50 \mathrm{mM}$ Tris- $\mathrm{HCl}$, $\mathrm{pH} 8.0,750 \mathrm{mM} \mathrm{KCl}, 2.5 \mathrm{mM}$ EDTA, $0.5 \%$ Triton-X, $62.5 \%$ glycerol and $1 \mathrm{mM} \mathrm{DTT})$. Increasing amounts of $\mathrm{Mg}^{2+}$ (10$1500 \mathrm{mM}$ ) or $0.1 \mathrm{nM}$ spermidine were added where appropriate. After $30 \mathrm{~min}$ at room temperature, the reaction mixture was applied to a $1.2 \%$ Tris-borate-EDTA (TBE)-agarose gel to resolve proteinDNA complexes from free DNA and visualized on a UV transilluminator after staining with ethidium bromide. Further analysis of the specificity and sensitivity of binding of BpeR to the bpeABoprB promoter in the presence of increasing spermidine concentrations (0.025-3.2 nM) was performed using the LightShift Chemiluminescent EMSA kit according to the manufacturer's protocol (Pierce). Briefly, $50 \mathrm{ng}$ purified BpeR was mixed with $50 \mathrm{ng}$ biotin-labelled DNA in binding buffer for $30 \mathrm{~min}$ at room temperature and then separated on a non-denaturing $5 \%$ polyacrylamide-TBE gel. The DNA was blotted onto a nylon membrane and detected using streptavidin-horseradish peroxidase (HRP) chemiluminescence (Pierce). Non-competitor DNA, comprising the $5^{\prime}$ end of the $b p e B$ gene, was PCR-amplified using the primer pair RTBpeBF (5'-AACGCGACGATCACGATCACA-3') and RT-BpeBR (5' TTCAGGTGCAGAACAAGCTGTCG- $3^{\prime}$ ). The specificity of the BpeR protein-bpeABoprB promoter binding in the mobility shift assay was ascertained by adding $1 \mu \mathrm{g}$ of a $78 \mathrm{bp}$ non-competitor DNA fragment to the binding reaction $10 \mathrm{~min}$ before the addition of labelled DNA target, and also by adding a 20 -fold excess $(1 \mu \mathrm{g})$ of unlabelled 
competitor (bpeABoprB promoter) DNA to the binding reaction 10 min prior to adding the labelled DNA target.

Accumulation of $\left[{ }^{14} \mathrm{C}\right]$ spermidine and $\left[{ }^{14} \mathrm{C}\right]$ erythromycin. The level of spermidine in wild-type $B$. pseudomallei KHW, the bpeAB mutant (KHW $\triangle$ bpeABKm) and the complemented bpeAB mutant (KHW $\triangle$ bpeABKm/pUCP28TbpeAB) was measured using the method described previously (Chan et al., 2004). Briefly, 16 h cultures of the bacteria in $5 \mathrm{ml} \mathrm{AB}$ medium containing $0.2 \%(\mathrm{w} / \mathrm{v})$ glucose and $0.2 \%$ casamino acids were washed three times in $\mathrm{AB}$ medium, and $100 \mu \mathrm{l}$ of the washed cells was inoculated into $2 \mathrm{ml}$ fresh $\mathrm{AB}$ medium containing $0.2 \%$ (w/v) glucose and $0.2 \%$ casamino acids. Dicyclohexylamine was added to a final concentration of $1 \mathrm{mM}$ to inhibit endogenous spermidine synthesis, and $\left[{ }^{14} \mathrm{C}\right]$ spermidine trichloride $\left[112 \mathrm{mCi} \mathrm{mmol}^{-1}\left(4.14 \mathrm{GBq} \mathrm{mmol}{ }^{-1}\right)\right.$ specific activity, Amersham Biosciences] was added to a final concentration of $20 \mathrm{nM}$. After incubating for $20 \mathrm{~h}$ at $37{ }^{\circ} \mathrm{C}$, an aliquot of the cell culture was removed for cell density measurement at $\mathrm{OD}_{600}$ and $500 \mu \mathrm{l}$ of the cell culture was harvested. The cells were washed three times in an equal volume of ice-cold $0.9 \%(w / v) ~ N a C l$ containing $20 \mathrm{nM}$ unlabelled spermidine. The cell pellet was air-dried and solubilized in $2 \mathrm{ml}$ CytoScint scintillation cocktail (MP Biomedicals) and counted using an LS6500 multipurpose scintillation counter (Beckman Instruments). The amount of $\left[{ }^{14} \mathrm{C}\right]$ spermidine retained was expressed as pmol per $10^{9}$ cells (i.e. picomoles per the number of cells in $1 \mathrm{OD}_{600}$ unit) and calibrated against a standard curve of 0-100 pmol $\left[{ }^{14} \mathrm{C}\right]$ spermidine. The experiment was performed in triplicate.

Accumulation of $\left[{ }^{14} \mathrm{C}\right]$ erythromycin was measured using intact $B$. pseudomallei KHW cells. Briefly, $100 \mu \mathrm{l}$ of a $16 \mathrm{~h}$ culture of $B$. pseudomallei $\mathrm{KHW}$ was inoculated into $5 \mathrm{ml} \mathrm{AB}$ medium containing $0.2 \%(\mathrm{w} / \mathrm{v})$ glucose and $0.2 \%$ casamino acids either with or without $1 \mathrm{mM}$ dicyclohexylamine. $\left[{ }^{14} \mathrm{C}\right]$ erythromycin $\left[47 \mathrm{mCi} \mathrm{mmol}^{-1}\right.$ $\left(1.74 \mathrm{GBq} \mathrm{mmol}^{-1}\right)$ specific activity, Perkin Elmer] was added to a final concentration of $0.1 \mu \mathrm{g} \mathrm{ml} \mathrm{g}^{-1}$ to the culture during early exponential phase $\left(\mathrm{OD}_{600} \sim 0.5\right)$. The MIC of erythromycin for $B$. pseudomallei KHW is $128 \mu \mathrm{g} \mathrm{ml}^{-1}$ (Chan et al., 2004). An aliquot of the culture, equivalent to $1 \mathrm{OD}_{600}$ unit, was removed at the start of the labelling and after 60 and $120 \mathrm{~min}$. The cell pellet was solubilized in $2 \mathrm{ml}$ CytoScint scintillation cocktail and the radioactivity counted as described above.

$\boldsymbol{\beta}$-Galactosidase assays. The assay for $\beta$-galactosidase was performed as described by Miller (1972). Briefly, $100 \mu \mathrm{l}$ of an overnight culture of $B$. pseudomallei KHW harbouring pCYYbpeAB was inoculated into $5 \mathrm{ml} \mathrm{AB}$ medium containing $0.2 \%(\mathrm{w} / \mathrm{v})$ glucose, $0.2 \%$ casamino acids and $25 \mu \mathrm{g}$ tetracycline $\mathrm{ml}^{-1}$. The culture was incubated at $37^{\circ} \mathrm{C}$ for $1 \mathrm{~h}$ with shaking at 100 r.p.m. to $\mathrm{OD}_{600} \sim 0.2$, after which spermidine or $\mathrm{N}$-acetylspermidine was added to give the final concentrations shown in Fig. 5. After $4 \mathrm{~h}$ incubation at $37^{\circ} \mathrm{C}$, the cell density $\left(\mathrm{OD}_{600}\right)$ was measured and $100 \mu \mathrm{l}$ of the culture was used for determining $\beta$-galactosidase activity. $\beta$-Galactosidase activity was expressed in Miller units and each assay was performed in triplicate.

Extraction and detection of radiolabelled $\left[{ }^{14} \mathrm{C}\right] \mathrm{AHLs}$ by RPHPLC. AHLs produced by B. pseudomallei KHW were radiolabelled, extracted and analysed by RP-HPLC, as described previously (Chan et al., 2007). The dried extract was dissolved in $200 \mu \mathrm{l} 20 \%$ (v/v) methanol and filtered through a $0.22 \mu \mathrm{m}$ pore-size membrane, and $100 \mu \mathrm{l}$ was injected into a $\mathrm{C}_{18}$ reversed-phase column for HPLC analysis. Fractions of $2 \mathrm{ml}$ were collected after elution at a flow rate of $1 \mathrm{ml} \mathrm{min}^{-1}$ using an isocratic profile of methanol: water $(50: 50, \mathrm{v} / \mathrm{v})$ for $10 \mathrm{~min}$, followed by a linear gradient of $50-90 \%$ methanol in water for $25 \mathrm{~min}$, and an isocratic profile of $90 \%$ methanol over $10 \mathrm{~min}$. Each fraction was dried, solubilized in $2 \mathrm{ml}$ CytoScint scintillation cocktail and counted using an LS6500 multipurpose scintillation counter. Peaks corresponding to $\mathrm{N}$ - octanoyl-homoserine lactone (C8HSL), N-decanoyl-homoserine lactone (C10HSL), 3-oxo-decanoyl-homoserine lactone (3-oxo-C10HSL), $\mathrm{N}$-3-oxo-tetradecanoyl-homoserine lactone (3-oxo-C14HSL), $\mathrm{N}$-3hydroxy-octanoyl-homoserine lactone (3-hydroxy-C8HSL), N-3hydroxy-decanoyl-homoserine lactone (3-hydroxy-C10HSL) and methionine were identified using $100 \mu \mathrm{l}$ of the standards $(1 \mathrm{mM})$, as described previously (Chan et al., 2007).

Swimming motility assay. Swimming motility was tested on LB medium containing $0.3 \%$ agar and different concentrations of dicyclohexylamine $(1,5$ or $10 \mathrm{mM})$ or methylthiopropylamine (1 or $5 \mathrm{mM}$ ). Two microlitres of a $16 \mathrm{~h}$ culture of B. pseudomallei KHW was inoculated into the centre of the agar and the plates were incubated at $30{ }^{\circ} \mathrm{C}$ for $20 \mathrm{~h}$. Control plates had no dicyclohexylamine or methylthiopropylamine.

Biofilm assay. Biofilm formation was measured as the amount of bacterial cells adhering to the wells of 96-well PVC microtitre plates, using a protocol modified from O’Toole \& Kolter (1998). Briefly, $100 \mu \mathrm{l}$ of a diluted overnight bacterial culture in LB medium $\left(\mathrm{OD}_{600}\right.$ of $~ 0.05)$ was added into each well of a 96-well PVC microtitre plate (Nunc). The wells contained different concentrations of dicyclohexylamine $(1,2,5$ and $10 \mathrm{mM})$ or methylthiopropylamine $(1,2$ and $5 \mathrm{mM}$ ). Control wells had no dicyclohexylamine or methylthiopropylamine, while blank wells had only LB medium. After incubating for $20 \mathrm{~h}$ at $37^{\circ} \mathrm{C}$, planktonic cells were removed and the wells washed twice with water. Adherent cells were stained with $125 \mu \mathrm{l} 1 \%(\mathrm{w} / \mathrm{v})$ crystal violet (Sigma-Aldrich) for $15 \mathrm{~min}$ at room temperature and then washed three times with water. The stain was dissolved in two aliquots of $100 \mu \mathrm{l} 95 \%(\mathrm{v} / \mathrm{v})$ ethanol and transferred to a fresh 96well plate to measure $A_{570}$. The assay was performed in triplicate.

\section{RESULTS}

\section{Growth phase changes in intracellular levels of polyamines and preferential extrusion of $\boldsymbol{N}$ - acetylspermidine}

Polyamines were extracted from $\sim 10^{9}$ (or $1 \mathrm{OD}_{600}$ unit) of B. pseudomallei KHW cells at different growth phases and analysed using RP-HPLC. Putrescine was the most abundant polyamine throughout the growth of $B$. pseudomallei KHW, and was at high intracellular levels $(260 \mathrm{pmol}$ putrescine per $10^{9}$ cells) even during early exponential phase $(6 \mathrm{~h})$. This was at least eightfold higher than the corresponding levels of spermidine ( 13 pmol per $10^{9}$ cells) and $\mathrm{N}$-acetylspermidine (30 pmol per $10^{9}$ cells). Although the amount of putrescine increased to $387 \mathrm{pmol}$ per $10^{9}$ cells in $24 \mathrm{~h}$ stationary phase cells, the change was not cell density-dependent (Fig. 1a). By comparison, the amounts of intracellular spermidine and $\mathrm{N}$-acetylspermidine were very low during the lag phase but increased according to cell density during the $\log$ and stationary phases. $\mathrm{N}$ Acetylspermidine levels also continued to increase in the bacterial cells even during the stationary phase, unlike spermidine, suggesting that spermidine was being converted to $\mathrm{N}$-acetylspermidine during the stationary phase (Fig. 1a). In spite of its abundance intracellularly, putrescine was not extruded into the culture medium. Only spermidine and $\mathrm{N}$-acetylspermidine were extruded by B. pseudomallei, and $\mathrm{N}$-acetylspermidine was the predom- 


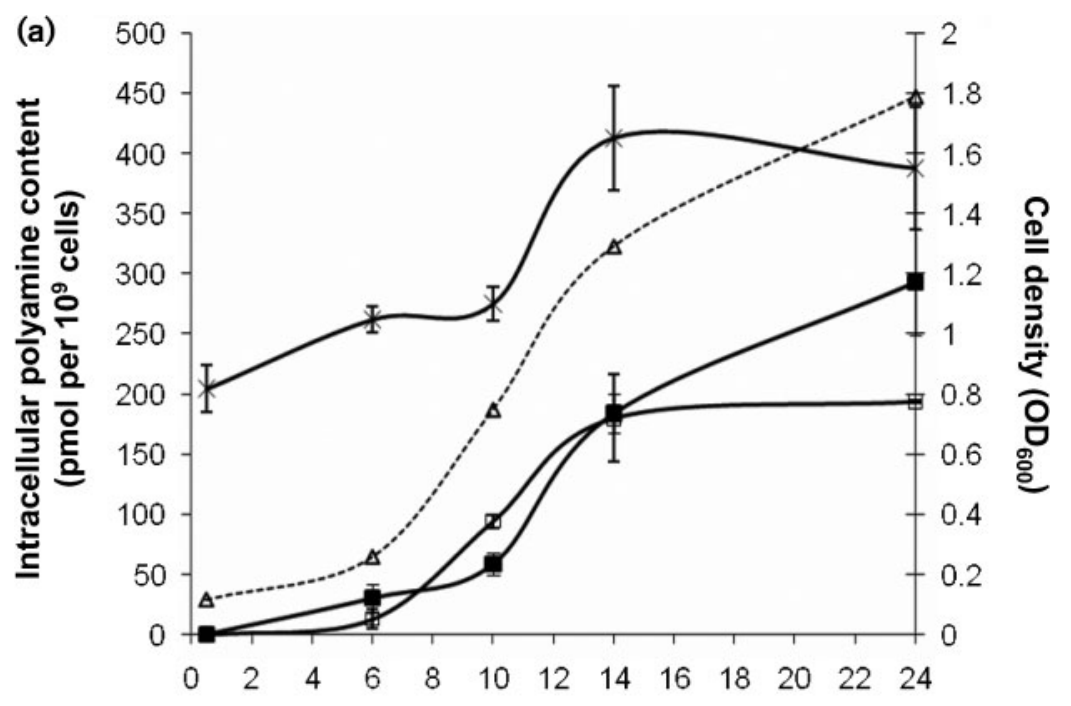

\title{
Duration of culture $(\mathrm{h})$
}

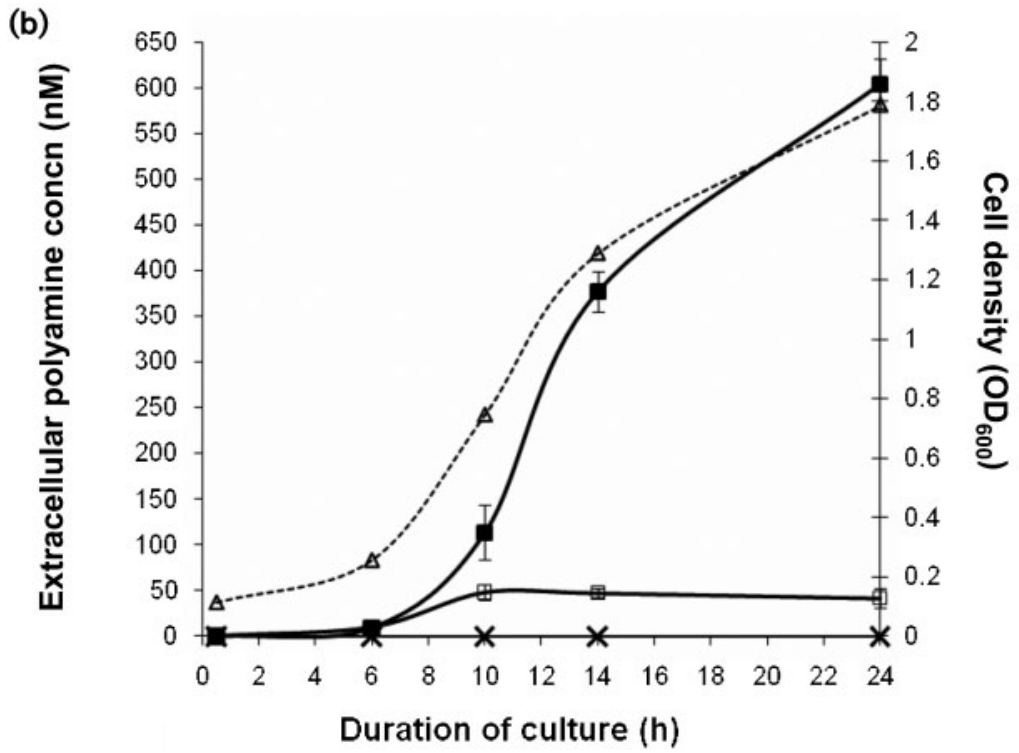

\begin{abstract}
Fig. 1. (a) Growth phase changes in intracellular polyamine content in wild-type B. pseudomallei $\mathrm{KHW}$ cultured in $\mathrm{AB}$ minimal medium containing $0.2 \%$ glucose. The polyamines were extracted from the cell pellet of $\sim 10^{9}$ (1 $\mathrm{OD}_{600}$ unit) B. pseudomallei KHW cells. (b) Growth phase changes in the extracellular concentrations of polyamines in wild-type $B$. pseudomallei $\mathrm{KHW}$. The polyamines were extracted from $1 \mathrm{ml}$ bacterial culture supernatant. Concentrations of extracellular polyamines were expressed as pmol ( $\mathrm{ml}$ culture medium $)^{-1}$ (or nM). $\triangle$, Cell density $\left(\mathrm{OD}_{600}\right) ; \times$, putrescine; 口, $N$-acetylspermidine; $\square$ spermidine. Values shown are the mean from three independent experiments; error bars, SD.
\end{abstract}

inant polyamine extruded (Fig. 1b). Extracellular concentrations of $\mathrm{N}$-acetylspermidine were 15 -fold higher than those of spermidine in $24 \mathrm{~h}$ cultures, although the changes in extracellular concentrations of both polyamines were clearly cell density-dependent.

\section{Involvement of the BpeAB-OprB pump in extrusion of $\boldsymbol{N}$-acetylspermidine and spermidine}

Evidence that the BpeAB-OprB pump is involved in the extrusion of polyamines was supported by data showing a fivefold reduction in extracellular $\mathrm{N}$-acetylspermidine concentration (123 nM) as well as undetectable levels of spermidine in the culture medium of the stationary phase (24 h) bpeAB mutant as compared with the wild-type (Fig. $2 \mathrm{~b})$. When exposed to radiolabelled $\left[{ }^{14} \mathrm{C}\right]$ spermidine, we also detected threefold higher retention of exogenous $\left[{ }^{14} \mathrm{C}\right]$ spermidine in the $b p e A B$ mutant after $20 \mathrm{~h}$ when compared with wild-type cells (Fig. 3). The level of exogenous $\left[{ }^{14} \mathrm{C}\right]$ spermidine in the complemented bpeAB mutant expressing $b p e A B$ from plasmid pUCP28T $b p e A B$ was reduced to almost wild-type levels (Fig. 3). We do not have data on the retention of exogenous radiolabelled $\mathrm{N}$-acetylspermidine, as the compound was not commercially available. It is also interesting to note the reduced levels of spermidine and $\mathrm{N}$ acetylspermidine in the $b p e A B$ mutant as compared with the wild-type (Figs 1a and 2a). The intracellular levels of spermidine and $N$-acetylspermidine in the bpeAB mutant at $24 \mathrm{~h}$ were three- to fourfold lower than the levels in the wildtype, although the intracellular levels of putrescine were unaffected. Although we attributed the reduction in the total amount of extracellular spermidine and $\mathrm{N}$-acetylspermidine 

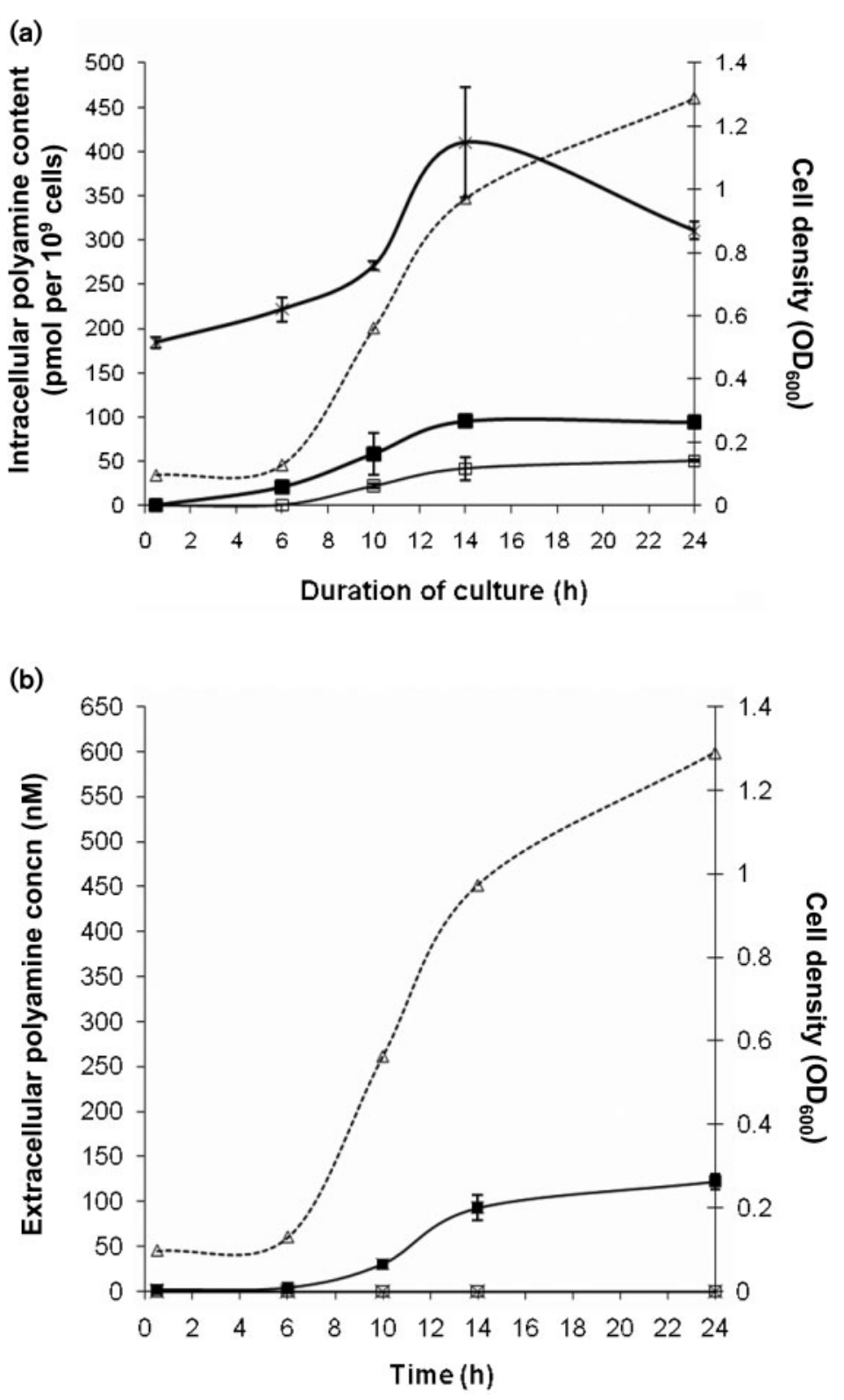

Fig. 2. (a) Changes in intracellular polyamine concentrations in the bpe $A B$ mutant during growth. Polyamines were extracted from the cell pellet of $\sim 10^{9}$ ( $1 \mathrm{OD}_{600}$ unit) $B$. pseudomallei KHW $\triangle b p e A B$ cells cultured in $\mathrm{AB}$ minimal medium containing $0.2 \%$ glucose. (b) Growth phase changes in extracellular concentrations of polyamines in the bpe $A B$ pump mutant. Polyamines were extracted from $1 \mathrm{ml}$ bacterial culture supernatant. The amounts of extracellular polyamines were expressed as nmol (I culture medium) $)^{-1} . \triangle$, Cell density $\left(\mathrm{OD}_{600}\right) ; \times$, putrescine; $\mathbf{\square}, \mathrm{N}$ acetylspermidine; $\square$, spermidine. Values shown are the mean from three independent experiments; error bars, SD. in the bpeAB mutant to an impaired efflux, the reduced intracellular levels of spermidine and $\mathrm{N}$-acetylspermidine in the bpeAB mutant suggest that an impaired BpeAB-OprB function also has a direct effect on spermidine biosynthesis. This effect seemed to be specific to the spermidine biosynthesis pathway, as the intracellular levels of putrescine were largely unaffected in the $b p e A B$ mutant.

\section{High levels of spermidine disrupt the binding of protein to DNA in vitro}

To determine the significance of changes in intracellular spermidine concentrations on gene expression, we used EMSA to show that increasing intracellular spermidine concentrations could disrupt the binding of regulatory proteins to DNA in vitro. Binding of the BpeR repressor protein to both the bpeABoprB promoter and the bpeR promoter was observed in the absence of spermidine but, in both instances, the binding was disrupted in the presence of $0.1 \mathrm{nM}$ spermidine (Fig. 4a, b). It was further verified using different concentrations of spermidine that the binding of the BpeR repressor to the bpeABoprB promoter occurred only if the spermidine concentration was $0.1 \mathrm{nM}$ or less, but not when the concentration was above $0.1 \mathrm{nM}$ (Fig. 4c). The EMSA experiment using a biotinylated DNA probe and chemiluminescent detection was significantly more sensitive than the method using ethidium bromide to detect shifted bands by agarose gel electrophoresis in Fig. 4(a), and could hence detect an additional higher-mobility shifted band in lanes 1 and 2 in Fig. 4(c). However, the lower shifted band 


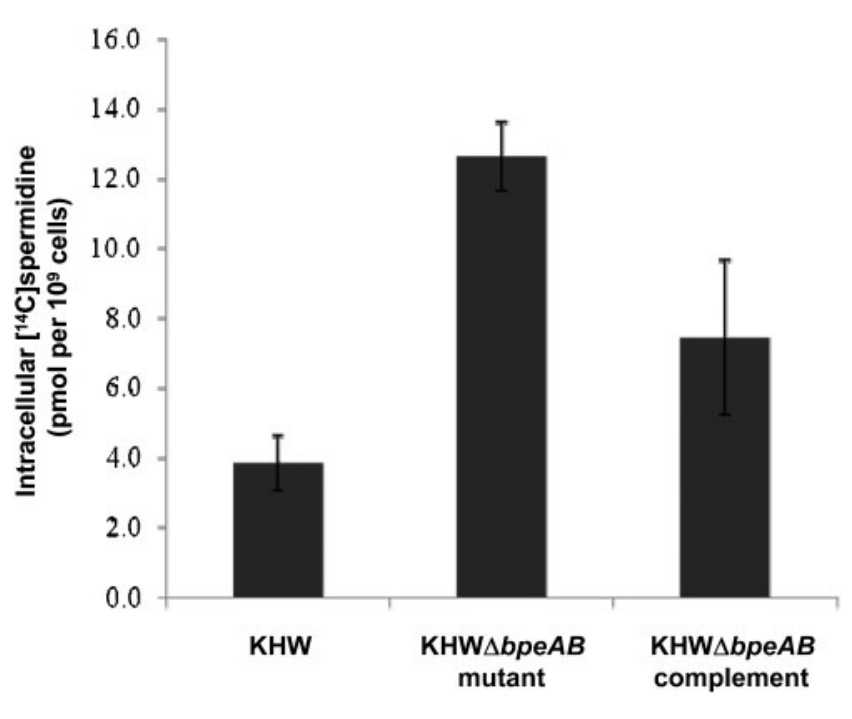

Fig. 3. Accumulation of exogenous $\left[{ }^{14} \mathrm{C}\right]$ spermidine in wild-type B. pseudomallei $\mathrm{KHW}$ cells, the bpeAB mutant and the complemented bpe $A B$ mutant. The amounts of $\left[{ }^{14} \mathrm{C}\right]$ spermidine in wild-type $B$. pseudomallei $\mathrm{KHW}$, the $\mathrm{KHW} \Delta b p e A B \mathrm{Km}$ mutant and the complemented KHW $\triangle b p e A B K m$ mutant were measured at $20 \mathrm{~h}$ after adding $20 \mathrm{nM}\left[{ }^{14} \mathrm{C}\right]$ spermidine and $1 \mathrm{mM}$ dicyclohexylamine to the culture medium. Values shown are the mean from three independent experiments; error bars, SD.

appeared to be non-specific, and was possibly due to the presence of non-specific contaminating His-tagged proteins or truncated BpeR protein in the protein preparation, since the upper band was the predominant shifted product in the presence of $0.1 \mathrm{nM}$ spermidine (lane 3 ). We also challenged the binding using excess non-competitor $b p e B$ DNA to ascertain specificity of the binding assay. BpeR did not bind to the non-competitor $b p e B$ DNA even in the absence of spermidine. Furthermore, excess unlabelled competitor bpeABoprB promoter DNA competed with the binding of BpeR to the labelled bpeABoprB promoter DNA in the absence of spermidine (Fig. 4c). Binding of BpeR repressor to the $b p e A B o p r B$ promoter could also be disrupted by gradually increasing the concentration of $\mathrm{Mg}^{2+}$ in the binding buffer from 10 to $100 \mathrm{mM}$, but the $\mathrm{Mg}^{2+}$ concentration needed to disrupt the protein-DNA interaction was at least $10^{5}$-fold higher than using spermidine (data not shown). We conclude that only small changes in intracellular spermidine concentration are sufficient to affect protein-DNA interaction.

\section{Exogenous spermidine and $\boldsymbol{N}$-acetylspermidine induce bpeA-lacZ expression in vivo in a dose- dependent manner}

Next, we showed that transcription of bpeA-lacZ in vivo could be induced by spermidine. Transcription of bpeAlac $Z$ was induced in a dose-dependent manner by the addition of exogenous spermidine to wild-type B. pseudo- mallei KHW harbouring plasmid pCYYbpeAB (Fig. 5a). Similar dose-dependent induction of $b p e A-l a c Z$ transcription was observed in the presence of exogenous $N$ acetylspermidine (Fig. 5b). A comparison of the induction levels of bpeA-lac $Z$ transcription in the presence of spermidine and $N$-acetylspermidine revealed that expression of bpeA-lac $Z$ was more sensitive to changes in concentrations of $N$-acetylspermidine, requiring $<1 \mathrm{nM}$ exogenous $\mathrm{N}$-acetylspermidine to achieve maximum induction as compared with $5 \mathrm{nM}$ exogenous spermidine (Fig. 5). Interestingly, no induction in the transcription of bpeA-lac $Z$ was observed in the presence of exogenous putrescine, even up to $50 \mathrm{nM}$ (data not shown).

\section{Inhibition of spermidine synthesis reduces efflux of $\left[{ }^{14}\right.$ C]erythromycin and AHLs}

Since spermidine could induce expression of bpeABoprB, we also explored the possibility of using spermidine synthase inhibitors to reduce the efflux of substrates by the BpeABOprB pump. Efflux of $\left[{ }^{14} \mathrm{C}\right]$ erythromycin was reduced by at least 4.5-fold in B. pseudomallei KHW cultured in the presence of $1 \mathrm{mM}$ dicyclohexylamine and a subinhibitory concentration $\left(0.1 \mu \mathrm{g} \mathrm{ml}{ }^{-1}\right)$ of $\left[{ }^{14} \mathrm{C}\right]$ erythromycin when compared with bacteria cultured in medium without dicyclohexylamine (Fig. 6). As the BpeAB-OprB pump is involved in the efflux of AHLs, dicyclohexylamine could also similarly reduce the efflux of AHLs and thus attenuate quorum sensing in B. pseudomallei KHW (Chan \& Chua, 2005). Analysis of ${ }^{14} \mathrm{C}$-labelled AHLs extracted from the culture supernatant of $24 \mathrm{~h} \mathrm{~B}$. pseudomallei KHW cultures revealed undetectable levels of all six AHLs, C8HSL, C10HSL, 3-hydroxy-C8HSL, 3-oxo-C10HSL, 3-hydroxyC10HSL and 3-oxo-C14HSL, when B. pseudomallei KHW was cultured in the presence of $1 \mathrm{mM}$ dicyclohexylamine (Fig. 7). Hence, spermidine synthase inhibitors can attenuate quorum sensing in B. pseudomallei KHW.

\section{Inhibition of spermidine synthesis reduces swimming motility and biofilm formation}

We also showed that intracellular spermidine levels can also affect swimming motility and biofilm formation in $B$. pseudomallei KHW. Swimming motility was progressively reduced in $B$. pseudomallei KHW cultured in LB medium containing 1,5 and $10 \mathrm{mM}$ dicyclohexylamine as well as $5 \mathrm{mM}$ methylthiopropylamine (Fig. 8a). Biofilm formation, a process which is dependent on bacterial motility, was also significantly reduced in B. pseudomallei KHW cultured in LB medium containing 1, 2, 5 and $10 \mathrm{mM}$ dicyclohexylamine, as well as in LB medium containing 1, 2 and $5 \mathrm{mM}$ methylthiopropylamine (Fig. 8b).

\section{DISCUSSION}

Stationary phase B. pseudomallei KHW produced higher levels of $N$-acetylspermidine than spermidine, although 


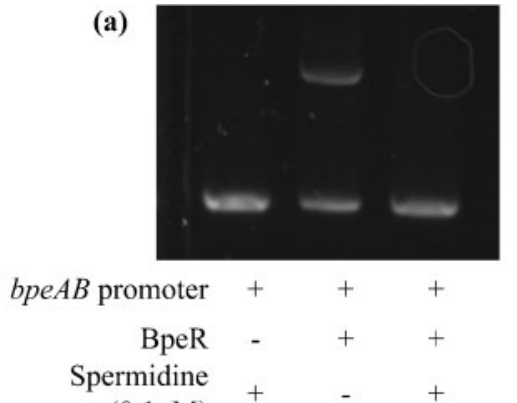

$(0 . \operatorname{lnM})$ (b)

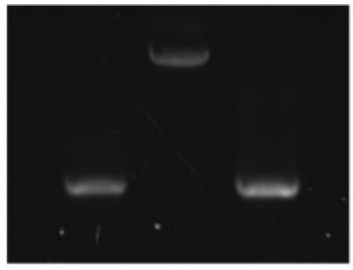

bpeR promoter $\quad+\quad+\quad+$

BpeR - ++

Spermidine

$(0.1 \mathrm{nM})$

(c)

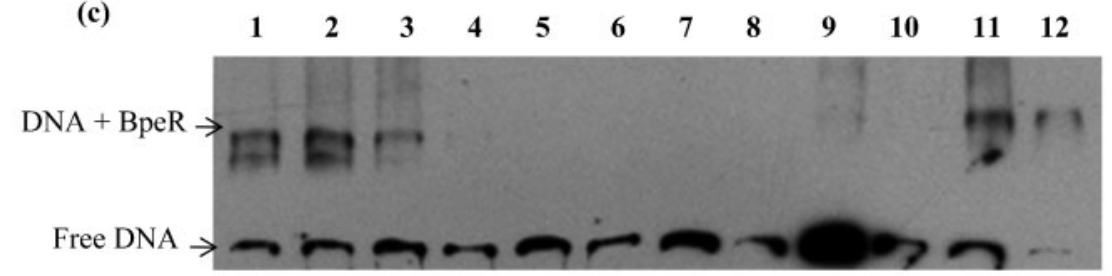

\begin{tabular}{|c|c|c|c|c|c|c|c|c|c|c|c|}
\hline $\begin{array}{r}\text { Labelled bpe } A B \\
\text { promoter }\end{array}$ & + & + & + & + & + & + & + & + & + & + & + \\
\hline $\begin{array}{r}\text { Unlabelled bpeAB } \\
\text { promoter }\end{array}$ & - & - & - & - & - & - & - & - & + & - & - \\
\hline Unlabelled $b p e B$ & - & - & - & - & - & - & - & - & - & - & + \\
\hline BpeR & + & + & + & + & + & + & + & + & + & - & + \\
\hline Spermidine (nM) & 0.025 & 0.05 & 0.1 & 0.2 & 0.4 & 0.8 & 1.6 & 3.2 & - & - & - \\
\hline
\end{tabular}

Fig. 4. EMSAs showing the effects of spermidine and $\mathrm{Mg}^{2+}$ concentrations on protein-DNA binding in vitro. In vitro binding of the BpeR protein to the bpeABoprB promoter (a) and the bpeR promoter (b) was disrupted in the presence of $0.1 \mathrm{nM}$ spermidine. (c) Titration of the in vitro binding of the BpeR protein to the bpeABoprB promoter with increasing concentrations of spermidine ranging from 0.025 to $3.2 \mathrm{nM}$. Lanes: $1-3$, binding of BpeR to the labelled bpeABoprB promoter at spermidine concentrations up to $0.1 \mathrm{nM} ; 4-8$, disruption of the BpeR-bpe $A B$ promoter binding at spermidine concentrations above $0.1 \mathrm{nM} ; 9$, disruption of the binding of BpeR protein to the labelled bpeABoprB promoter by excess unlabelled competitor bpe $A B$ oprB promoter; 10 , unbound labelled bpe $A B$ oprB promoter alone; 11, no competition in the binding of BpeR to the labelled bpe $A B$ opr $B$ promoter in the presence of excess unlabelled non-competitor bpeB DNA; 12 , binding of BpeR to the labelled bpeABoprB promoter in the absence of spermidine.

the levels of both increased significantly during the growth of the bacteria. Conversion of spermidine to $\mathrm{N}$ acetylspermidine seems to be a response to stress, such as limiting nutrients or the oxidative stress associated with entry to stationary phase, as observed in E. coli cells (Carper et al., 1991). Unlike spermidine, $\mathrm{N}$-acetylspermidine is physiologically inert and does not bind to RNA or stimulate in vitro translation. Therefore, active conversion of spermidine to $\mathrm{N}$-acetylspermidine by spermidine acetyltransferase would be an effective mechanism to reduce the toxicity associated with high levels of spermidine (Kakegawa et al., 1991). Although speculative, it is possible that cell density-dependent changes in intracellular spermidine levels, which are linked to methionine utilization and the production of $S$-adenosylmethionine (SAM), function as a metabolic sensor for $B$. pseudomallei to regulate gene expression and protein synthesis in a growth phase-dependent manner. Spermine, which is present in eukaryotic cells, has not been detected in B. pseudomallei KHW and other bacteria (Igarashi \& Kashiwagi, 2000).

In B. pseudomallei KHW, acetylation seems to serve not only to detoxify spermidine but also to facilitate its extrusion from the bacterium. An E. coli speG mutant lacking spermidine acetyltransferase shows growth inhibition during cold shock due to the accumulation of high levels of spermidine. The growth inhibition is attributed to ribosome inactivation caused by a replacement of ribosome-bound $\mathrm{Mg}^{2+}$ by spermidine (Limsuwun \& Jones, 2000). The E. coli speG mutant is also sensitive to exogenous spermidine and exhibits decreased cell viability, especially when exogenous spermidine is added to $24 \mathrm{~h}$ stationary phase cells (Fukuchi et al., 1995). It is possible 

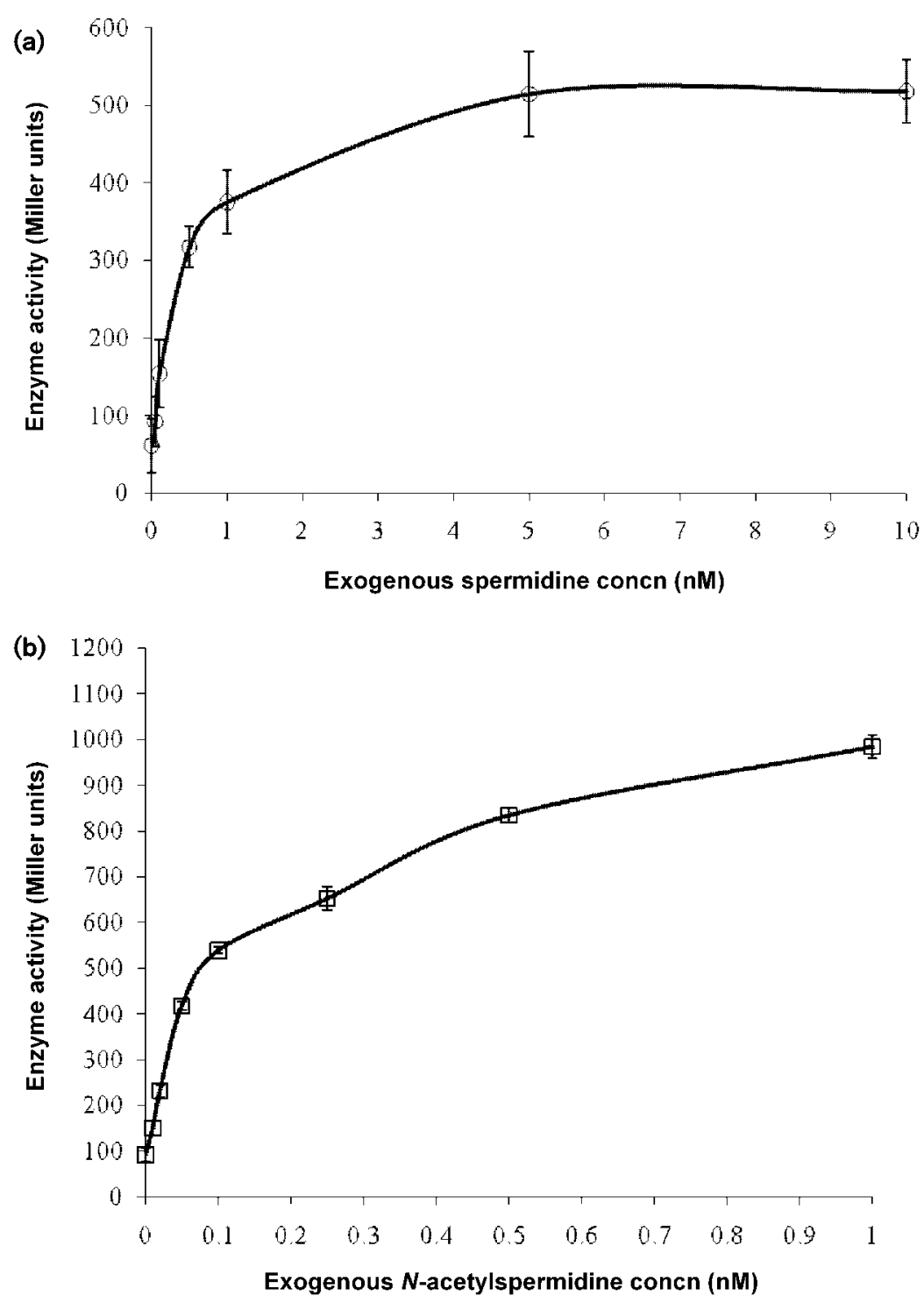

\begin{abstract}
Fig. 5. Induction of bpeA-lacZ expression in $B$. pseudomallei $\mathrm{KHW}$ by exogenous spermidine and $N$-acetylspermidine. (a) Dose-dependent induction of bpeA-lacZ expression in the presence of increasing concentrations of exogenous spermidine

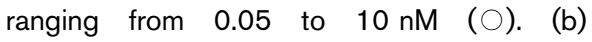
Dose-dependent induction of bpeA-lacZ expression in the presence of increasing concentrations of exogenous $\mathrm{N}$-acetylspermidine ranging from 0.01 to $1 \mathrm{nM}(\square)$. Values shown are the mean from three independent experiments; error bars, SD.
\end{abstract}

that spermidine in $B$. pseudomallei is also actively acetylated to overcome the inhibition of protein synthesis that might otherwise occur during stationary phase. It has also been noted that bacteria such as E. coli do not have any spermidine deacetylating activity or polyamine oxidase activity, so $N$-acetylspermidine cannot be recycled but has to be extruded from the cell. Our data support the view that spermidine is actively acetylated and extruded from B. pseudomallei as $N$-acetylspermidine by the BpeABOprB pump, although some of this inert polyamine remained in the cells during stationary phase. A plausible explanation for the preferential efflux of acetylated spermidine by the BpeAB-OprB pump would be a change in the structural properties of spermidine at physiological $\mathrm{pH}$ that reduces its binding affinity to the pump when compared with $\mathrm{N}$-acetylspermidine (Karahalios et al., 1998). We also noted the presence of low levels of $N$ acetylspermidine $(\sim 20 \%$ of wild-type levels $)$ in the extracellular medium of the $b p e A B$ mutant and attribute this to an involvement of a secondary mechanism other than BpeAB-OprB in its efflux.

Together with $\mathrm{Mg}^{2+}$, polyamines account for the majority of the intracellular cationic charges, and they are essential for normal cell growth and viability (Canellakis et al., 1979). Although the effect of polyamines on cell growth is believed to occur at the level of translation, we have shown that polyamines can also regulate gene expression at the transcriptional level in vitro by affecting the binding of regulatory proteins to DNA. The polyamine modulon in $E$. coli has been defined as a group of genes whose expression is regulated by polyamines and comprises several transcription factors whose translation is affected by polyamines (Igarashi \& Kashiwagi, 2006). We have shown that small changes in spermidine concentration affect the binding of the BpeR repressor protein to the operator regions of the bpeABoprB and $b p e R$ promoters in vitro. The ability of spermidine to induce $b p e A-l a c Z$ transcription in 


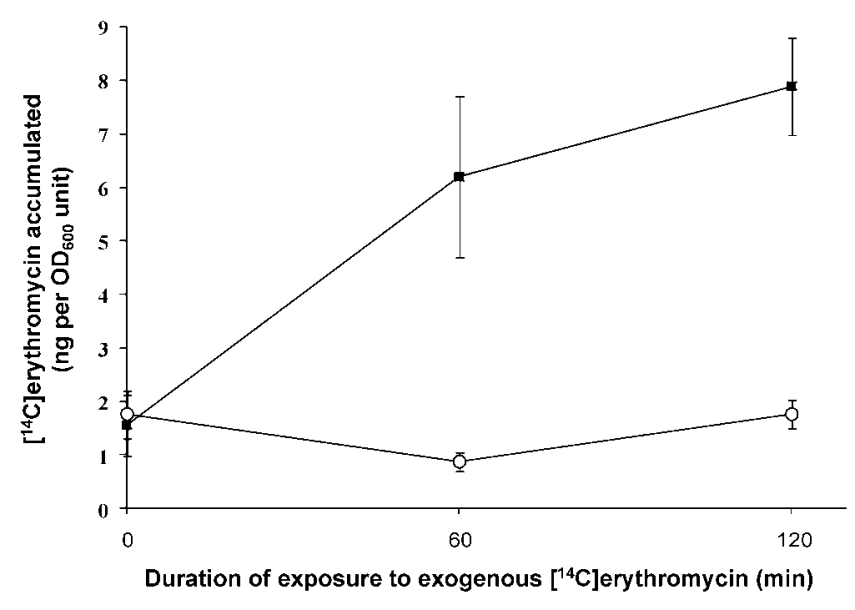

Fig. 6. Dicyclohexylamine increases the retention of $\left[{ }^{14} \mathrm{C}\right]$ erythromycin in B. pseudomallei KHW cells. $\mathbf{~}$, B. pseudomallei $\mathrm{KHW}$ cells cultured in $A B$ medium containing $0.2 \%(w / v)$ glucose and $1 \mathrm{mM}$ exogenous dicyclohexylamine; $\bigcirc$, B. pseudomallei $\mathrm{KHW}$ cells cultured in $A B$ medium containing $0.2 \%(w / v)$ glucose alone. Values shown are the mean from three independent experiments; error bars, SD.

vivo in a dose-dependent manner supports the in vitro data. It is also possible that changes in intracellular spermidine affect expression of many other genes.

We propose that spermidine, which is polycationic, interacts with the binding of BpeR to DNA in the same manner that $\mathrm{Mg}^{2+}$ affects protein-DNA interaction, albeit with much higher sensitivity. Therefore, depending on the metabolic state of the bacteria, subtle changes in intracellular spermidine concentrations may be sufficient to mediate changes in gene expression in B. pseudomallei KHW. Using dicyclohexylamine to inhibit intracellular spermidine synthesis, we showed that efflux of $\left[{ }^{14} \mathrm{C}\right]$ erythromycin by the BpeAB-OprB pump could be reduced significantly, thus uncovering a mechanism for modulating efflux pump activity. Real-time PCR transcriptional analysis of eight putative RND efflux pumps in stationary phase $B$. pseudomallei KHW cultured in $\mathrm{AB}$ medium containing dicyclohexylamine revealed significant downregulation in the transcription of all the pumps, including BpeAB-OprB, AmrAB-OprB and BpeEF-OprC (data not shown).

Due to the involvement of BpeAB-OprB in the efflux of AHLs, we showed that inhibiting intracellular spermidine synthesis can also block the extracellular production of AHLs required for quorum sensing in B. pseudomallei. Optimal intracellular spermidine concentrations are also required for cellular functions of $B$. pseudomallei $\mathrm{KHW}$ other than cell growth. Inhibition of intracellular spermidine synthesis also reduced biofilm formation and swimming motility in B. pseudomallei KHW. It is possible that some of these phenotypes can be attributed to a direct effect of spermidine on the BpeAB-OprB pump, which has a significant impact on antibiotic resistance, quorum sensing, biofilm formation and virulence when impaired (Chan \& Chua, 2005). Dicyclohexylamine also inhibits the synthesis of spermidine and growth in $P$. aeruginosa, accompanied by inhibition of motility and synthesis of flagella, while increasing concentrations of norspermidine in Vibrio cholera activate biofilm formation (Karatan et al., 2005; Paulin et al., 1986). Considering the importance of quorum sensing in $B$. pseudomallei virulence and biofilm formation, spermidine synthase inhibitors could be novel anti-infective and anti-biofilm agents against $B$. pseudomallei (Chan \& Chua, 2005).

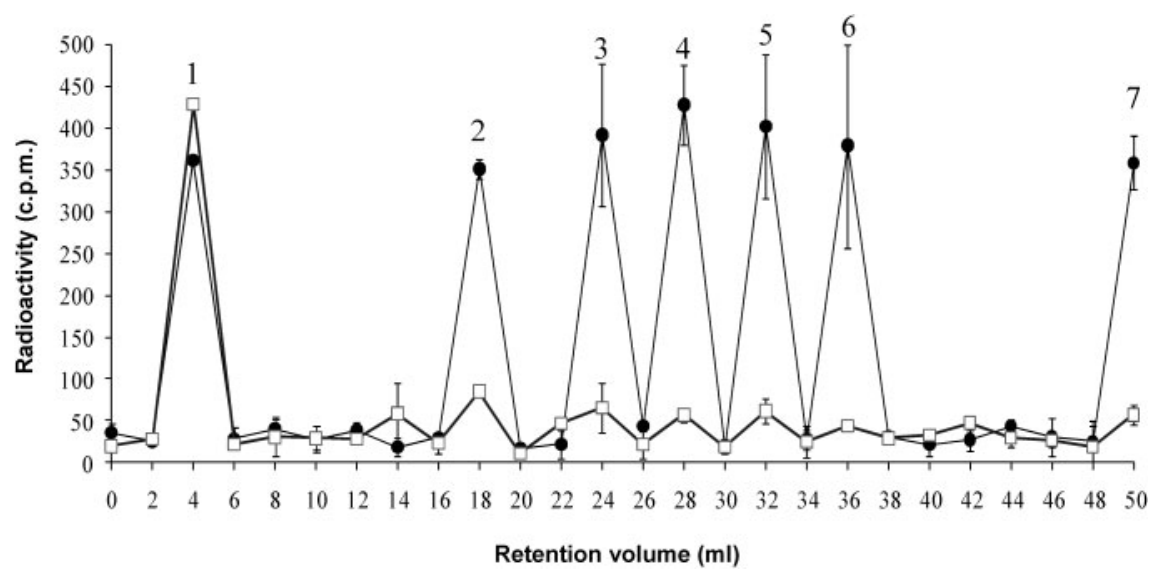

Fig. 7. Dicyclohexylamine attenuates quorum sensing by inhibiting production of extracellular AHLs. Analysis of extracellular AHLs extracted from stationary phase $(24 \mathrm{~h}) \mathrm{B}$. pseudomallei KHW cultured in AB medium only ( $\bullet$ ) and in $A B$ medium containing $1 \mathrm{mM}$ dicyclohexylamine ( $\square$ ). Peak positions 1, 2, 3, 4, 5, 6 and 7 correspond to the elution profiles of methionine, C8HSL, C10HSL, 3-hydroxy-C8HSL, 3-oxo-C10HSL, 3-hydroxy-C10HSL and 3-oxo-C14HSL, respectively. Values shown are the mean from three independent experiments; error bars, SD. 
(a)
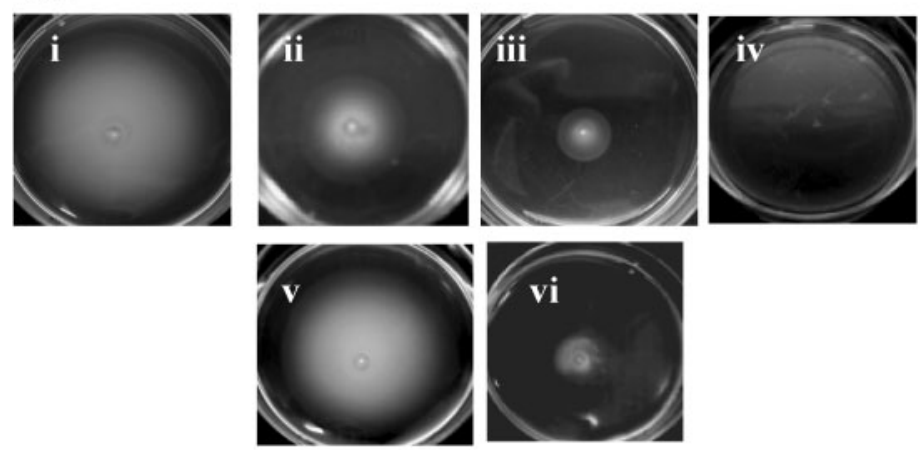

(b)

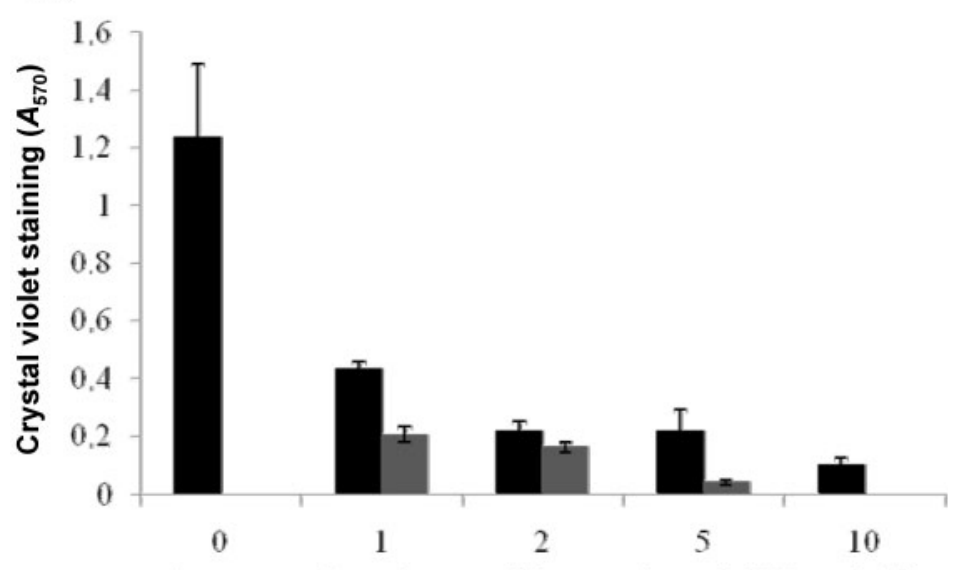

Concentration of spermidine synthase inhibitor ( $\mathrm{mM}$ )

Fig. 8. Effect of spermidine on B. pseudomallei and biofilm formation. (a) Swimming motility of $B$. pseudomallei KHW was reduced in LB medium containing $0.3 \%$ agar and increasing concentrations of spermidine synthase inhibitors. (i) LB medium only; (ii-iv) with 1, 5 and $10 \mathrm{mM}$ dicyclohexylamine; (v, vi) with 1 and $5 \mathrm{mM}$ methylthiopropylamine. (b) $B$. pseudomallei KHW formed less biofilm in LB medium containing increasing concentrations of spermidine synthase inhibitors. Black and grey bars show amounts of biofilm formed in the presence of dicyclohexylamine and methylthiopropylamine, respectively. Values shown are the mean from four independent experiments; error bars, SD.

\section{ACKNOWLEDGEMENTS}

This work was supported by a grant from the National Medical Research Council of Singapore (NMRC/1012/2005).

\section{REFERENCES}

Canellakis, E. S., Viceps-Madore, D., Kyriakidis, D. A. \& Heller, J. S. (1979). The regulation and function of ornithine decarboxylase and of the polyamines. Curr Top Cell Regul 15, 155-202.

Carper, S. W., Willis, D. G., Manning, K. A. \& Gerner, E. W. (1991). Spermidine acetylation in response to a variety of stresses in Escherichia coli. J Biol Chem 266, 12439-12441.

Chan, Y. Y. \& Chua, K. L. (2005). The Burkholderia pseudomallei BpeAB-OprB efflux pump: expression and impact on quorum sensing and virulence. J Bacteriol 187, 4707-4719.

Chan, Y. Y., Tan, T. M., Ong, Y. M. \& Chua, K. L. (2004). BpeAB-OprB, a multidrug efflux pump in Burkholderia pseudomallei. Antimicrob Agents Chemother 48, 1128-1135.

Chan, Y. Y., Bian, H. S., Tan, T. M., Mattmann, M. E., Geske, G. D., Igarashi, J., Hatano, T., Suga, H., Blackwell, H. E. \& Chua, K. L. (2007). Control of quorum sensing by a Burkholderia pseudomallei multidrug efflux pump. J Bacteriol 189, 4320-4324.

Cheng, A. C. \& Currie, B. J. (2005). Melioidosis: epidemiology, pathophysiology, and management. Clin Microbiol Rev 18, 383-416.

Clark, D. \& Maaløe, O. (1967). DNA replication and the division cycle in Escherichia coli. J Mol Biol 23, 99-112.
Fried, M. \& Crothers, D. M. (1981). Equilibria and kinetics of lac repressor-operator interactions by polyacrylamide gel electrophoresis. Nucleic Acids Res 9, 6505-6525.

Fukuchi, J., Kashiwagi, K., Yamagishi, M., Ishihama, A. \& Igarashi, K. (1995). Decrease in cell viability due to the accumulation of spermidine in spermidine acetyltransferase-deficient mutant of Escherichia coli. J Biol Chem 270, 18831-18835.

Ha, H. C., Sirisoma, N. S., Kuppusamy, P., Zweier, J. L., Woster, P. M. \& Casero, R. A., Jr (1998). The natural polyamine spermine functions directly as a free radical scavenger. Proc Natl Acad Sci U S A 95, 11140-11145.

Huang, S. C., Panagiotidis, C. A. \& Canellakis, E. S. (1990). Transcriptional effects of polyamines on ribosomal proteins and on polyamine-synthesizing enzymes in Escherichia coli. Proc Natl Acad Sci U S A 87, 3464-3468.

Hwang, D. F., Chang, S. H., Shiua, C. Y. \& Chai, T. (1997). Highperformance liquid chromatographic determination of biogenic amines in fish implicated in food poisoning. J Chromatogr $B$ Biomed Sci Appl 693, 23-29.

Igarashi, K. \& Kashiwagi, K. (2000). Polyamines: mysterious modulators of cellular functions. Biochem Biophys Res Commun 271, 559-564.

Igarashi, K. \& Kashiwagi, K. (2006). Polyamine modulon in Escherichia coli: genes involved in the stimulation of cell growth by polyamines. J Biochem 139, 11-16.

Jung, I. L. \& Kim, I. G. (2003a). Transcription of $a h p C$, $k a t G$, and $k a t E$ genes in Escherichia coli is regulated by polyamines: polyaminedeficient mutant sensitive to $\mathrm{H}_{2} \mathrm{O}_{2}$-induced oxidative damage. Biochem Biophys Res Commun 301, 915-922. 
Jung, I. L. \& Kim, I. G. (2003b). Polyamines and glutamate decarboxylase-based acid resistance in Escherichia coli. J Biol Chem 278, 22846-22852.

Kakegawa, T., Guo, Y., Chiba, Y., Miyazaki, T., Nakamura, M., Hirose, S., Canellakis, Z. N. \& Igarashi, K. (1991). Effect of acetylpolyamines on in vitro protein synthesis and on the growth of a polyamine-requiring mutant of Escherichia coli. J Biochem 109, 627-631.

Karahalios, P., Mamos, P., Vynios, D. H., Papaioannou, D. \& Kalpaxis, D. L. (1998). The effect of acylated polyamine derivatives on polyamine uptake mechanism, cell growth, and polyamine pools in Escherichia coli, and the pursuit of structure/activity relationships. Eur J Biochem 251, 998-1004.

Karatan, E., Duncan, T. R. \& Watnick, P. I. (2005). NspS, a predicted polyamine sensor, mediates activation of Vibrio cholerae biofilm formation by norspermidine. J Bacteriol 187, 7434-7443.

Khan, A. U., Di Mascio, P., Medeiros, M. H. \& Wilson, T. (1992). Spermine and spermidine protection of plasmid DNA against singlestrand breaks induced by singlet oxygen. Proc Natl Acad Sci U S A 89, 11428-11430.

Kumar, A., Chua, K. L. \& Schweizer, H. P. (2006). Method for regulated expression of single-copy efflux pump genes in a surrogate Pseudomonas aeruginosa strain: identification of the BpeEF-OprC chloramphenicol and trimethoprim efflux pump of Burkholderia pseudomallei 1026b. Antimicrob Agents Chemother 50, 3460-3463.

Kwon, D. H. \& Lu, C. D. (2006). Polyamines induce resistance to cationic peptide, aminoglycoside, and quinolone antibiotics in Pseudomonas aeruginosa PAO1. Antimicrob Agents Chemother 50, 1615-1622.

Limsuwun, K. \& Jones, P. G. (2000). Spermidine acetyltransferase is required to prevent spermidine toxicity at low temperatures in Escherichia coli. J Bacteriol 182, 5373-5380.
Miller, J. H. (1972). Experiments in Molecular Genetics. Cold Spring Harbor, NY: Cold Spring Harbor Laboratory.

Moore, R. A., DeShazer, D., Reckseidler, S., Weissman, A. \& Woods, D. E. (1999). Efflux-mediated aminoglycoside and macrolide resistance in Burkholderia pseudomallei. Antimicrob Agents Chemother 43, 465-470.

O'Toole, G. A. \& Kolter, R. (1998). Initiation of biofilm formation in Pseudomonas fluorescens WCS365 proceeds via multiple, convergent signalling pathways: a genetic analysis. Mol Microbiol 28, 449-461.

Paulin, L., Lindberg, L. A. \& Poso, H. (1986). Reversible inhibition of flagella formation after specific inhibition of spermidine synthesis by dicyclohexylamine in Pseudomonas aeruginosa. Antonie Van Leeuwenhoek 52, 483-490.

Pearson, J. P., Van Delden, C. \& Iglewski, B. H. (1999). Active efflux and diffusion are involved in transport of Pseudomonas aeruginosa cell-to-cell signals. J Bacteriol 181, 1203-1210.

Pegg, A. E., Bitonti, A. J., McCann, P. P. \& Coward, J. K. (1983). Inhibition of bacterial aminopropyltransferases by $S$-adenosyl-1,8-diamino-3thiooctane and by dicyclohexylamine. FEBS Lett 155, 192-196.

Seiler, N. (1987). Functions of polyamine acetylation. Can J Physiol Pharmacol 65, 2024-2035.

Tabor, C. W. \& Tabor, H. (1985). Polyamines in microorganisms. Microbiol Rev 49, 81-99.

White, N. J. (2003). Melioidosis. Lancet 361, 1715-1722.

Yoshida, M., Meksuriyen, D., Kashiwagi, K., Kawai, G. \& Igarashi, K. (1999). Polyamine stimulation of the synthesis of oligopeptidebinding protein (OppA). Involvement of a structural change of the Shine-Dalgarno sequence and the initiation codon AUG in Oppa mRNA. J Biol Chem 274, 22723-22728.

Edited by: P. Cornelis 